\title{
Cardiac Memory: A Rare Cause of Postoperative Deep T Wave Inversions
}

\author{
Ankit B. Shah ${ }^{\mathrm{a}, \mathrm{b}, \mathrm{c}}$, Matthew Katz ${ }^{\mathrm{a}, \mathrm{b}}$, Stavros E. Mountantonakis ${ }^{\mathrm{a}, \mathrm{b}}$
}

\begin{abstract}
Cardiac memory $(\mathrm{CM})$ is a phenomenon of electrical remodeling seen after periods of altered ventricular conduction. In this unique finding, the T wave direction during "memory", or periods after altered depolarization, is similar to that of the QRS complex during periods of abnormal depolarization. The $\mathrm{T}$ wave abnormalities are commonly seen after ventricular pacing, but can also been seen with intermittent left bundle branch block (LBBB). We present a case of a high-risk elderly male who developed deep T wave inversions (TWIs) postoperatively. While ischemia is highest on the differential diagnosis in such a case, our patient was asymptomatic with negative serial troponins. Also, our patient developed the deep TWIs in the setting of an intermittent LBBB. The diagnosis of CM was made after thorough evaluation of other causes of TWIs. It is important for clinicians to be aware of CM, as it is often misinterpreted as ischemia and early diagnosis can help prevent unnecessary invasive workup.
\end{abstract}

Keywords: Cardiac memory; Electrocardiography; T wave inversion; Intermittent left bundle branch block

\section{Introduction}

First coined by Rosenbaum et al in 1982, cardiac memory $(\mathrm{CM})$ is a phenomenon of electrical remodeling seen after periods of altered ventricular conduction [1]. In this unique finding, the T wave direction during "memory", or periods after altered depolarization, is similar to that of the QRS complex during periods of abnormal depolarization. Such $\mathrm{T}$ wave abnormalities have been seen after termination of ventricular

Manuscript accepted for publication October 08, 2015

aDepartment of Cardiology, Lenox Hill Heart and Vascular Institute of New York, New York, NY, USA

${ }^{b}$ Hofstra North Shore-LIJ School of Medicine at Hofstra University, Hempstead, NY, USA

${ }^{\mathrm{c} C o r r e s p o n d i n g ~ A u t h o r: ~ A n k i t ~ B . ~ S h a h, ~ L e n o x ~ H i l l ~ H o s p i t a l, ~} 100$ East 77th St., 2nd Floor Non-Invasive Cardiology, New York, NY 10075, USA.

Email: shahcsmc@gmail.com

doi: http://dx.doi.org/10.14740/jmc2333w pacing, elimination of ventricular pre-excitation after ablation of an accessory pathway and temporary resolution of conduction abnormalities (predominantly left bundle branch block (LBBB)) [1]. We present a case of a high-risk elderly male who developed deep T wave inversions (TWIs) postoperatively in the setting of intermittent LBBB. A discussion of the differential diagnosis of TWIs and the differentiation of $\mathrm{CM}$ from ischemia follow.

\section{Case Report}

A 93-year-old man with a history of diabetes and coronary artery disease with prior coronary artery bypass graft developed sinus bradycardia with new ST-T wave changes after endovascular repair of an abdominal aortic aneurysm. The patient was hemodynamically stable and asymptomatic.

The patient's baseline electrocardiogram (ECG) (Fig. 1) demonstrated normal sinus rhythm, first degree atrioventricular (AV) block, LBBB, TWIs in I, aVL, v5 and v6 and atrial premature depolarizations. The postoperative ECG (Fig. 2) demonstrates sinus bradycardia at 48 beats/min and first degree AV block with resolution of LBBB. There are voltage criteria consistent with left ventricular hypertrophy (LVH) and notably TWIs in all leads, and particularly pronounced in the precordial leads.

An echocardiogram done postoperatively showed LVH with an ejection fraction of $25 \%$ and an akinetic apex and anteroseptal wall (unchanged from prior). Serial troponins were negative $(<0.015 \mathrm{ng} / \mathrm{mL})$. On postoperative day 1 , the patient was no longer bradycardic resulting in reinstitution of his baseline ECG with LBBB, and resolution of the deep TWIs. The patient remained asymptomatic throughout his hospitalization and was discharged 2 days later.

\section{Discussion}

In an elderly patient with extensive atherosclerotic disease, ischemia of the proximal LAD was highest on the differential diagnosis as the cause of new deep precordial TWIs (Wellens' sign) [2]. Other causes of TWIs include electrolytes abnormalities (especially hypokalemia), hypertrophic cardiomyopathy, hypothyroidism, pulmonary embolism, arrhythmogenic right 


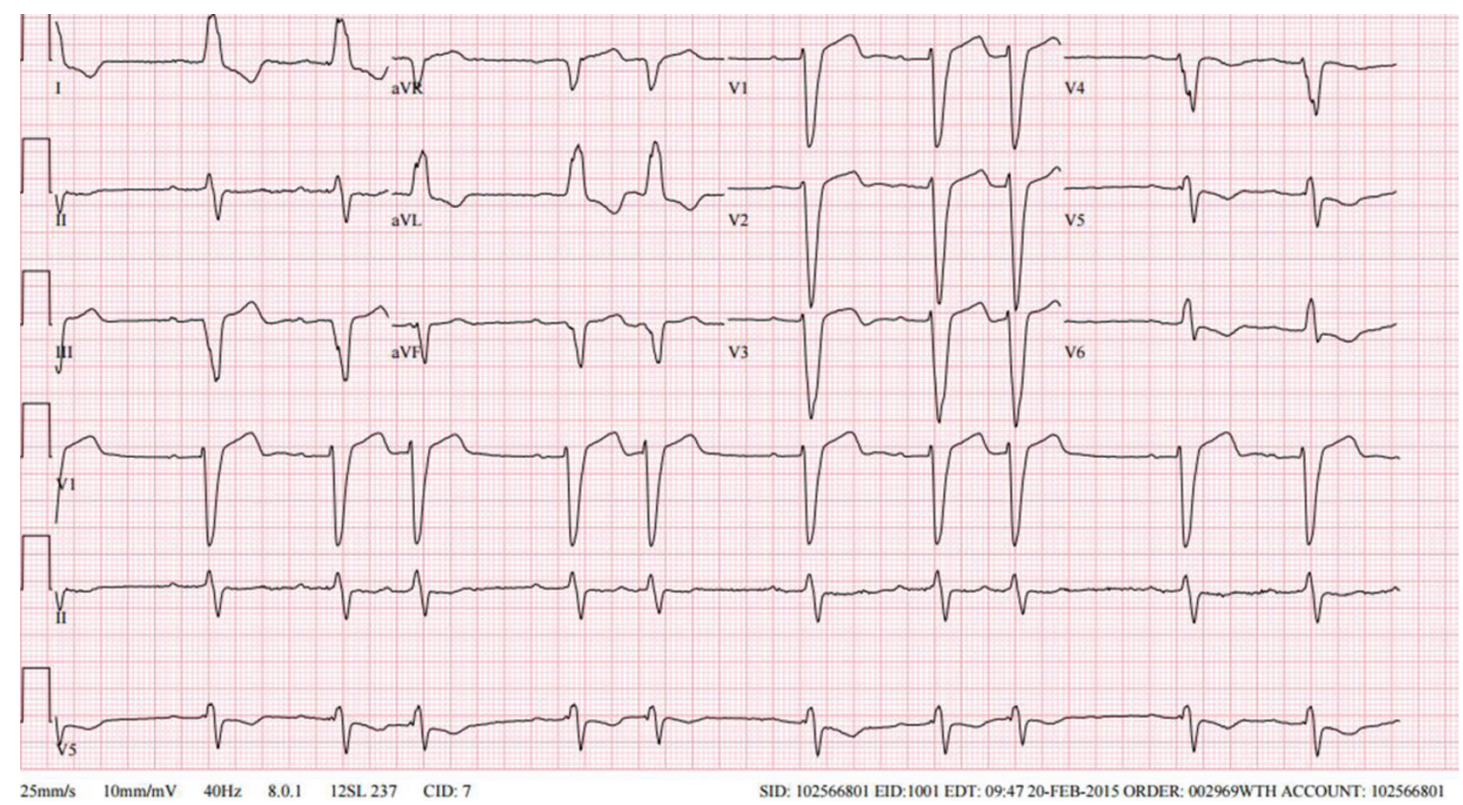

Figure 1. Baseline ECG.

ventricular cardiomyopathy, persistent juvenile T waves, medications and an intracranial process (particularly hemorrhage).

Another possible explanation for the aforementioned ECG changes is the phenomenon of CM. While this is a diagnosis of exclusion, the deep TWI in the setting of temporary resolution of LBBB, negative serial troponins and absence of any other clinical explanation are strongly suggestive. Typically, the presence of a bundle branch block during physiologic heart rates and resolution at lower heart rates suggests significant but not complete anatomical disruption of that bundle. As the Purkinje fibers conduction is governed by the "all or none" principle, with no effect of the autonomic nervous system, there is a heart rate threshold over which all electrical impulses are blocked. This threshold decreases as the Purkinje network becomes more diseased [3]. This should be distinguished from the presence of right bundle branch block at higher heart rates, which is a normal functional phenomenon and a result of the refractory period of the right bundle branch being longer than

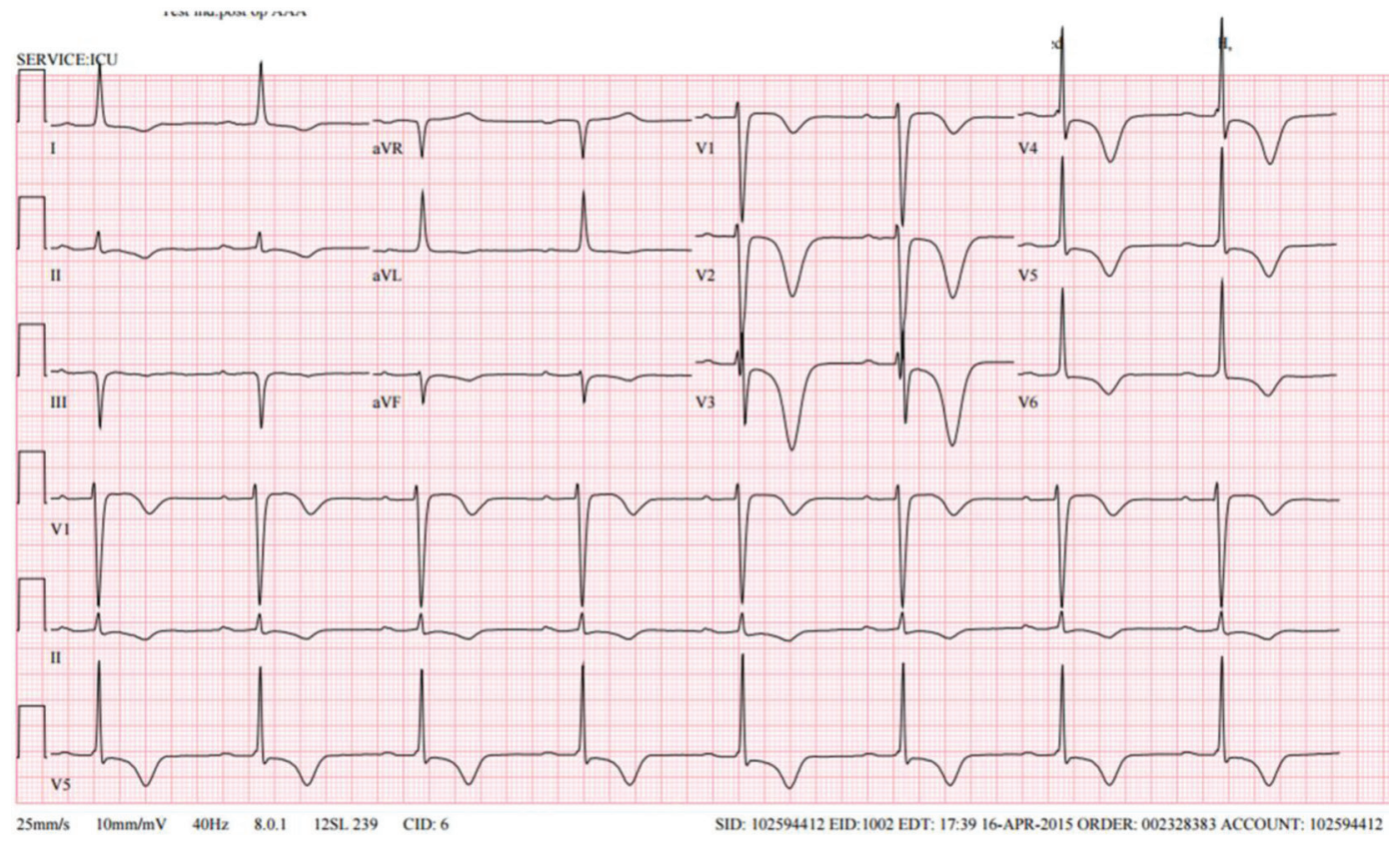

Figure 2. Postoperative ECG from current presentation. 
that of the left bundle branch.

$\mathrm{CM}$ poses a challenge in ECG interpretation, as it often imitates findings of ischemia. Distinguishing $T$ wave changes in CM from those in ischemia is important, as correctly identifying $\mathrm{CM}$ can avoid unnecessary additional invasive testing. The presence of a positive $\mathrm{T}$ wave in aVL, a positive or isoelectric $\mathrm{T}$ wave in lead I, and a maximal precordial TWI that is greater than the TWI in lead III, is $92 \%$ sensitive and $100 \%$ specific for CM as suggested in a cohort of 60 patients [4]. Our patient was known to have structural heart disease and therefore, baseline ST-T changes are expected and thus, the TWIs seen in Figure 1 are likely due to LVH or an old infarct. Existing data in patients with structural heart disease suggest that when TWIs are present in leads I and aVL at baseline, they can remain inverted even with the development of CM $[5]$.

\section{Conclusion}

This case demonstrates the complexities that CM can add to the interpretation of ECGs in a high-risk patient. While criteria exist to help to distinguish CM ECG changes from ischemic changes, there can be confounding data and the diagnosis requires a thorough evaluation of other causes of TWI. Most importantly, $\mathrm{T}$ wave memory remains a diagnosis of exclusion, and therefore, history, clinical presentation and review of prior ECGs remain the foundation of clinical decision-making.

\section{Disclosures}

There are no relevant financial disclosures, acknowledgments or conflicts of interest. All authors had access to the data and a role in writing the manuscript.

\section{References}

1. Rosenbaum MB, Blanco HH, Elizari MV, Lazzari JO, Davidenko JM. Electrotonic modulation of the $\mathrm{T}$ wave and cardiac memory. Am J Cardiol. 1982;50(2):213-222.

2. de Zwaan C, Bar FW, Wellens HJ. Characteristic electrocardiographic pattern indicating a critical stenosis high in left anterior descending coronary artery in patients admitted because of impending myocardial infarction. Am Heart J. 1982;103(4 Pt 2):730-736.

3. Josephson ME. Intraventricular Conduction Disturbances. In: Josephson ME, ed. Clinical Cardiac Electrophysiology: Techniques and Interpretation. 4th ed. Philadelphia, PA: Lippincott Williams \& Wilkins; 2008:114-144.

4. Shvilkin A, Ho KK, Rosen MR, Josephson ME. T-vector direction differentiates postpacing from ischemic T-wave inversion in precordial leads. Circulation. 2005;111(8):969-974.

5. Shvilkin A, Huang HD, Josephson ME. Cardiac memory: diagnostic tool in the making. Circ Arrhythm Electrophysiol. 2015;8(2):475-482. 